\title{
3D Simulations of Ion Thruster Accelerator Grid Erosion Accounting for Charge Exchange Ion Space Charge
}

\author{
Vernon H. Chaplin*, James E. Polk ${ }^{\dagger}$, Ira Katz ${ }^{\ddagger}$, and John R. Anderson ${ }^{\S}$ \\ NASA Jet Propulsion Laboratory, California Institute of Technology, Pasadena, CA, 91109, USA \\ George J. Williams Jr. ${ }^{\text {Il }}$, George C. Soulas" , and John T. Yim** \\ NASA Glenn Research Center, Cleveland, $\mathrm{OH}, 44135$, USA
}

\begin{abstract}
Accelerator (accel) grid sputtering by ions formed through charge-exchange (CEX) reactions between beam ions and residual neutral gas is a critical life-limiting mechanism for gridded ion thrusters. The three-dimensional ion optics code CEX3D is designed to simulate this grid erosion for a single beamlet, with a particular emphasis on non-axisymmetric features such as the "pits and grooves" erosion commonly observed on the accel grid downstream face in two-grid thrusters. The treatment of CEX ions in the code was recently upgraded with a new particle-in-cell (PIC) module to account for the influence of these ions' space charge on the electrostatic potential downstream of the grids. In order to achieve reasonable computation times while resolving the Debye length near the grids and avoiding gross violations of the Courant-Friedrichs-Lewy (CFL) condition, macroparticle velocities in the PIC calculation are limited through a rescaling procedure that preserves ion trajectories and space charge density. The code accounts for beam divergence, finite momentum transfer in CEX collisions, and radial losses of CEX ions from the beam; these effects are important for determining the CEX ion flux to the accel grid because the calculated potential downstream of the grids can become very flat. The upgraded code has been used to simulate operation of NASA's Evolutionary Xenon Thruster (NEXT) during the $51 \mathrm{kHr}$ Long Duration Test-a selection of results is presented and compared with experimental data.
\end{abstract}

\section{Nomenclature}

$\begin{array}{lll}\mathrm{CEX} & =\text { charge exchange } \\ \lambda_{D e} & =\text { electron Debye length } \\ \epsilon_{0} & =\text { vacuum permittivity } \\ k_{B} & =\text { Boltzmann constant } \\ T_{e} & =\text { electron temperature } \\ n_{e} & =\text { electron density } \\ e & =\text { electron charge } \\ \phi & =\text { electrostatic potential } \\ \phi_{0}, n_{e 0} & =\text { potential and electron density at a reference location } \\ \mathrm{TL} & =\text { throttle level } \\ d_{S} & =\text { screen grid hole diameter } \\ z & =\text { axial direction in the beamlet coordinate system } \\ x & =\text { transverse coordinate in the direction toward the nearest adjacent beamlet } \\ \dot{n}_{C E X} & =\text { CEX ion creation rate per unit volume } \\ n_{\text {beam }} & =\text { beam ion density } \\ \end{array}$

\footnotetext{
*Technologist, Electric Propulsion Group, 4800 Oak Grove Dr., Pasadena, CA 91109, AIAA Member

${ }^{\dagger}$ Principle Engineer, Propulsion and Materials Engineering Section, 4800 Oak Grove Dr., Pasadena, CA 91109, AIAA Associate Fellow

${ }^{\ddagger}$ Principle Engineer, Propulsion and Materials Engineering Section, 4800 Oak Grove Dr., Pasadena, CA 91109, AIAA Senior Member

${ }^{\S}$ Senior Engineer, Contamination Control Engineering Group, 4800 Oak Grove Dr., Pasadena, CA 91109, AIAA Member

II Research AST, Electric Propulsion Systems Branch, 21000 Brookpark Rd., Cleveland, OH 44135, AIAA Associate Fellow

"Aerospace Engineer, Electric Propulsion Systems Branch, 21000 Brookpark Rd., Cleveland, OH 44135, AIAA Member

***esearch Engineer, Electric Propulsion Systems Branch, 21000 Brookpark Rd., Cleveland, OH 44135
} 


\begin{tabular}{|c|c|c|}
\hline$\sigma_{C E X}$ & $=$ & cross section for charge exchange \\
\hline$E_{\text {beam }}$ & $=$ & beam ion energy \\
\hline$v_{\text {beam }}$ & $=$ & beam ion velocity \\
\hline$n_{\text {gas }}$ & $=$ & neutral gas density \\
\hline$M_{i}$ & $=$ & ion mass \\
\hline$\phi_{\text {upstream }}$ & $=$ & potential at upstream domain boundary \\
\hline$T_{e, \text { upstream }}$ & $=$ & electron temperature at upstream domain boundary \\
\hline E & $=$ & ion energy, or electric field \\
\hline $\mathrm{Xe}^{+}$ & $=$ & singly ionized xenon \\
\hline $\mathrm{Xe}^{2+}$ & $=$ & doubly ionized xenon \\
\hline$n_{\text {gas, upstream }}$ & $=$ & neutral gas density at upstream domain boundary \\
\hline$C$ & $=$ & Clausing factor \\
\hline$\delta$ & $=$ & downstream gas density correction factor \\
\hline$D(r, z, a)$ & $=$ & Disk Gas function \\
\hline$h$ & $=$ & ratio of accel grid hole area to beamlet cross sectional area \\
\hline$z_{\text {accel }}$ & $=$ & axial position of accel grid downstream face \\
\hline$r_{\text {accel }}$ & $=$ & accel grid hole radius \\
\hline vipstream & $=$ & thruster neutral gas mean axial velocity at upstream domain boundary \\
\hline$v_{z, \text { gas }}^{\text {downstream }}$ & $=$ & thruster neutral gas mean axial velocity at downstream domain boundary \\
\hline$\theta_{1}, \theta_{2}, \theta_{1}$ & $=$ & angles defined in Disk Gas function \\
\hline$p(\theta)$ & $=$ & probability density for CEX scattering at angle $\theta$ \\
\hline$A_{C E X}$ & $=$ & power law fit parameter for CEX scattering angle \\
\hline$v_{C E X}$ & $=$ & velocity gained by the CEX ion (formerly a neutral) in a CEX collision \\
\hline$\Delta t$ & $=$ & time step in PIC simulation \\
\hline$P$ & $=$ & probability for CEX ion macroparticle creation, or facility background pressure \\
\hline$V_{\text {cell }}$ & $=$ & computational cell volume \\
\hline$q_{\min }$ & $=$ & minimum charge for a CEX ion macroparticle in the PIC calculation \\
\hline $\mathbf{x}_{n}, \mathbf{v}_{n}$ & $=$ & ion position and velocity at PIC time step $n$ \\
\hline $\mathbf{E}_{n}$ & $=$ & electric field at time step $n$ \\
\hline$x_{\max }$ & $=$ & domain length in the $x$ direction \\
\hline$y_{\max }$ & $=$ & domain length in the $y$ direction \\
\hline$\Delta x$ & $=$ & distance between mesh nodes \\
\hline$V_{b}$ & $=$ & beam voltage \\
\hline$q$ & $=$ & macroparticle charge \\
\hline$m$ & $=$ & macroparticle mass \\
\hline$K$ & $=$ & kinetic energy \\
\hline$\gamma$ & $=$ & macroparticle velocity rescaling factor \\
\hline$\rho$ & $=$ & charge density \\
\hline$v_{\max }$ & $=$ & threshold for velocity rescaling \\
\hline$v_{x}, v_{y}, v_{z}$ & $=$ & macroparticle velocity in $x, y$, and $z$ directions \\
\hline$\gamma_{1}, \gamma_{2}$ & $=$ & partial velocity rescaling factors \\
\hline$J$ & $=$ & current density \\
\hline$f$ & $=$ & Courant-Friedrichs-Lewy (CFL) limit multiplier \\
\hline$I_{a}$ & $=$ & accel grid current \\
\hline$I_{b}$ & $=$ & beamlet or beam current \\
\hline$R_{\text {optics }}$ & $=$ & grid radius of curvature \\
\hline$z_{b}$ & $=$ & birth location for a CEX ion \\
\hline$j_{b}$ & $=$ & CEX ion creation rate per unit volume \\
\hline$R$ & $=$ & ratio of beam voltage to total accelerating voltage \\
\hline
\end{tabular}

\section{Introduction}

CCelerator (accel) grid erosion is a key life-limiting mechanism for gridded ion thrusters. Well-designed ion Aoptics allow little or no direct impingement of beam ions on the grids, but charge exchange (CEX) collisions 
between beam ions and neutral gas produce slow CEX ions that are attracted toward the negatively biased accel grid and sputter it. Erosion occurs primarily on the grid aperture walls ("barrel erosion") and on the downstream face, where the hexagonal hole layout of typical ion thruster grids leads to a characteristic "pits and grooves" erosion pattern [1].

Computational modeling of the beam and CEX ion dynamics is a valuable tool for ion thruster design and for predicting the flight service life of existing thrusters. Between the screen and accel grids and inside the apertures, individual beamlets are nearly cylindrically symmetric, so 2D beamlet simulations can effectively model barrel erosion. On the other hand, pits and grooves erosion is fundamentally non-axisymmetric and thus must be treated in 3D.

The codes CEX2D and CEX3D, so named because they track CEX ion birth rates and trajectories and thus can predict grid erosion rates due to impacts by these ions, were originally developed at NASA Jet Propulsion Laboratory (JPL) roughly 16 years ago [2, 3], and additional features were subsequently added [4, 5] to improve the codes' ability to predict thruster performance and lifetime. A number of other 2D [6-8] and 3D [9-14] ion optics models have also been developed and used to simulate thruster performance and grid erosion. Many of these computations have focused on three-grid ion optics, for which accel grid downstream face erosion is limited and barrel erosion is the primary concern for lifetime. Others addressed pits and grooves erosion by postulating the location of a neutralization surface downstream of the grids beyond which CEX ions do not flow back upstream, or by empirically adjusting the injected CEX ion current to match the accel grid current measured in ground tests. However, predicting the flight service life of two-grid ion optics requires a self-consistent, 3D model of the CEX ion population and electrostatic potential extending $\gg 1 \mathrm{~cm}$ downstream of the grids. In this paper, we report on recent upgrades to JPL's CEX3D code geared toward achieving this predictive capability.

The work described here was carried out primarily to support analysis of the NASA Evolutionary Xenon Thruster (NEXT) Long Duration Test (LDT) which took place from 2005 to 2014 at NASA Glenn Research Center [15]. Interpretion of the results of this wear test has been complicated by the significant quantity of carbon facility material back-sputtered onto the grids during thruster operation [16]; thus there was an opportunity for simulations of both the LDT itself as well as NEXT operation under space vacuum to make valuable contributions to the flight service life assessment.

The main new feature in CEX3D is a particle-in-cell (PIC) module that self-consistently accounts for the effect of CEX ion space charge on the potential. Although this space charge is a small perturbation on the beam ion charge density, we will show that it has a critical effect on the slope of the potential profile downstream of the grids, which ultimately determines the size of the region from which CEX ions can flow back to the grids and cause erosion. In Sec. III], we introduce the main features of the CEX3D code and provide background on its status at the start of this project. Sec. IV describes the new PIC CEX ion model and other key code upgrades in detail. Simulation predictions are compared with results from the NEXT LDT in Sec.V] Finally, Sec.VI discusses conclusions and suggests a path forward for service life modeling of two-grid ion optics.

\section{Background on CEX3D}

CEX3D [3] simulates a single ion thruster beamlet while incorporating boundary conditions that account for ions and neutral gas traveling between beamlets. Properties of the discharge chamber plasma (ion density, electron temperature, and gas temperature) are used as boundary inputs for the ion optics calculation-the computational domain begins a few hole diameters upstream of the screen grid and typically extends downstream 5-10 cm. In order to save computation time, the code simulates a 30-60-90 triangle spanning 1/12 of the beamlet-hexagonal symmetry implies that the full beamlet solution can be obtained by reflections and rotations of the solution for this wedge volume [12]. The mesh volumes that make up the domain are right triangular prisms. Variable mesh spacing is used in the axial direction, with progressively larger mesh volumes in the downstream region where the ion density is low. In order to obtain accurate potential solutions and ion trajectories, the mesh spacing near the grids must typically be no larger than $\sim 1 / 2$ of the electron Debye length, $\lambda_{D e} \equiv \sqrt{\epsilon_{0} k_{B} T_{e} / n_{e} e^{2}}$. This restriction is less important in the downstream region, where the potential gradients are not as steep and beam ions are already traveling at high velocities.

The code first solves for beam ion trajectories in the self-consistent potential created by the grid biases and space charge, then calculates CEX ion creation rates and trajectories. Macroparticles representing beam ions are pushed through a static potential, with charge density assigned to each node in proportion to the time each macroparticle spends in its vicinity (this is sometimes known as a "flux tube" approximation [12, 14]), then the potential solution is updated and the process is repeated until convergence. Since electrons move much faster than xenon ions, their density is assumed to follow the Boltzmann relation, $n_{e}(\phi)=n_{e 0} \exp \left(e\left(\phi-\phi_{0}\right) / k_{B} T_{e}\right)$, where different electron temperatures and reference densities and potentials are used for the regions upstream and downstream of the grids. The nonlinear 


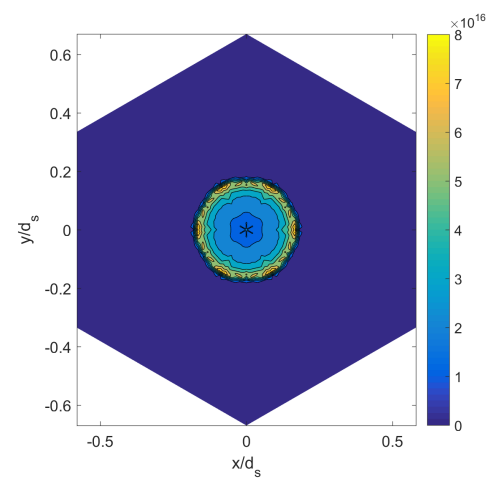

(a)

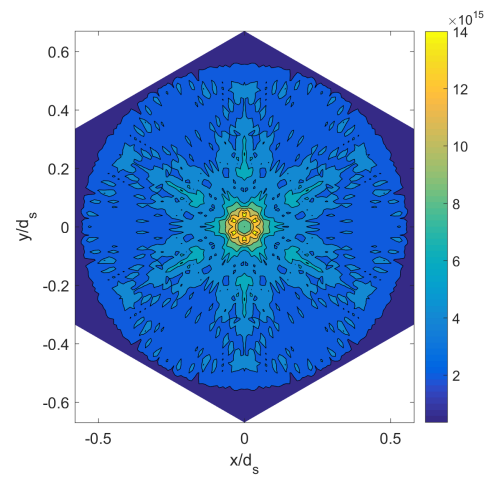

(b)

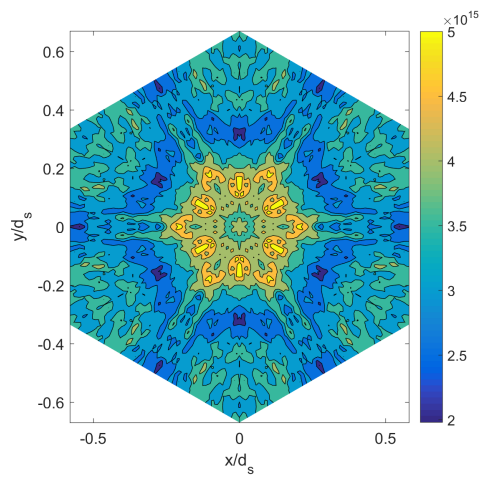

(c)

Fig. 1 Simulated beam ion density cross sections at axial distances 0 (a), 3.6 (b), and 8.8 (c) screen grid hole diameters downstream of the accel. grid, for the maximum beamlet current at the $679 \mathrm{~V}, 1.2 \mathrm{~A}$ NEXT operating condition (throttle level TL5).

Poisson's equation resulting from this expression is linearized using a Taylor expansion,

$$
n_{e}\left(\phi_{\text {new }}\right) \approx n_{e, \text { old }}+\left.\frac{\partial n_{e}}{\partial \phi}\right|_{\phi_{\text {old }}}\left(\phi_{\text {new }}-\phi_{\text {old }}\right)=n_{e, \text { old }}+\frac{e}{k_{B} T_{e}} n_{e, \text { old }}\left(\phi_{\text {new }}-\phi_{\text {old }}\right),
$$

and the resulting matrix equation is solved to find the potential $\phi_{\text {new }}$ everywhere on the mesh. The potential solver was recently upgraded to utilize the PARDISO parallel sparse direct solver [17, 18], accessed through the Intel Math Kernel Library (MKL). In order to ensure that the potential only changes slightly from one iteration to the next so that the first-order Taylor expansion is valid, the upstream beam ion density is initialized at a low value and gradually built up to obtain the actual beamlet current. Figures 1 and 2 show examples of converged beam ion density solutions, representative beam ion trajectories, and potentials calculated by CEX3D. Distances in all figures are normalized to the minimum screen grid hole diameter $d_{s}$.

The neutral gas distribution near the grids is calculated using an analytical model for the gas density near a disk-shaped source or sink, supplemented by a Monte Carlo calculation of the Clausing factor [1] for an approximate grid geometry. After obtaining the ion beam solution, the code calculates the CEX ion creation rate in every mesh volume and then follows macroparticles representing these ions until they strike a grid or cross the upstream or downstream boundary of the domain. The local sputtering rate due to CEX and beam ion impacts is recorded at each node within the grids.

Previous versions of CEX3D attempted to account for CEX ion space charge in a computationally efficient manner by pushing macroparticles through a static potential until they reached a grid or left the domain, updating the potential based on the macroparticles' charge and residence time near each node, and then iterating until a steady state potential was reached. While this approach works well for beam ions, for CEX ions the calculation did not always converge. These slow ions would follow meandering trajectories through the region downstream of the grids and could get stuck in small potential wells set up by the beam ion density distribution. Since the potential was not self-consistently updated at every particle-pushing time step, unphysical ion densities could build up in the wells, rendering the calculation unstable. Therefore, it was clear that a standard particle-in-cell (PIC) approach [19] was required.

\section{Particle-in-cell Model for CEX Ions}

The new PIC module for the CEX ions includes the following basic steps:

1) Calculate the time step for particle pushing.

2) Birth CEX ion macroparticles based on the charge exchange rate in each mesh volume.

3) Advance CEX ion velocities and positions by one time step, rescale macroparticle velocity, charge, and mass if necessary, and update the CEX ion space charge on each node.

4) Kill CEX particles that have hit a grid, traveled past the downstream end of the domain, or left the beam radially. 




(a)

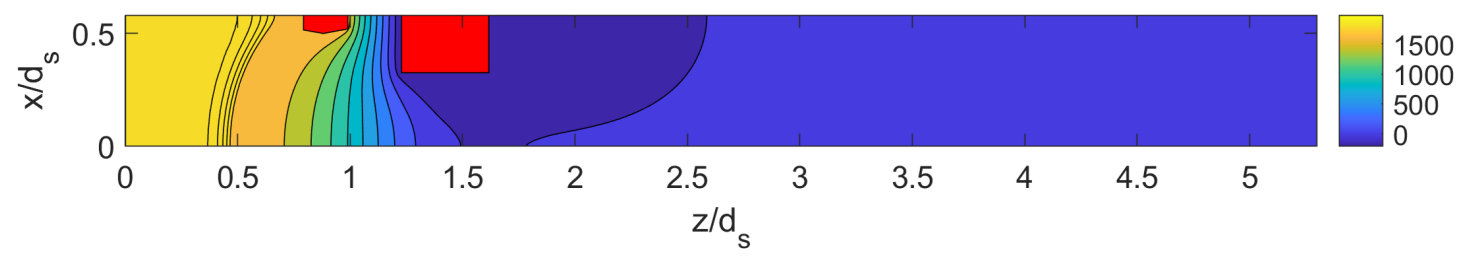

(b)

Fig. 2 Simulated beam ion trajectories (a) and potential contours produced by beam ions alone (b) in the $x-z$ plane (where $\hat{z}$ is the axial direction and $\hat{x}$ is the direction toward the nearest adjacent beamlet), for the maximum beamlet current at the $1800 \mathrm{~V}, 3.52$ A NEXT operating condition (TL40). Only $\sim 10 \%$ of the actual domain length is shown.

5) Calculate current densities and erosion rates on grid surfaces.

6) Solve for the potential throughout the domain.

7) Repeat for a number of iterations specified by the user (usually until steady state is reached).

The code enters this loop following the completion of the beam ion trajectory and potential calculation. The beam ion charge density and the neutral gas density are held fixed during the CEX ion PIC calculation (the beam is not attenuated to account for charge exchange, which only changes its density slightly over the length scales of the interest for the simulations). Changes in the downstream region potential on the order of $\pm 1 \mathrm{~V}$ caused by CEX ion space charge do not affect the beam ion trajectories significantly since they have energies $\gg 100 \mathrm{~V}$ in this region. In the following sections, we will describe the implementation of the calculation steps enumerated above in detail.

\section{A. CEX Ion Creation}

The CEX ion creation rate per unit volume is

$$
\dot{n}_{C E X}=n_{\text {beam }}\left\langle\sigma_{C E X}\left(E_{\text {beam }}\right) v_{\text {beam }}\right\rangle n_{\text {gas }} .
$$

All beam ions have approximately the same velocity at a given position:

$$
v_{\text {beam }}(\mathbf{x})=\sqrt{\frac{2 e}{M_{i}}\left(\phi_{\text {upstream }}-\phi(\mathbf{x})+\frac{T_{e, \text { upstream }}}{2}\right)} .
$$

We use the cross section formula from Ref. [20]:

$$
\sigma_{C E X}(E)=87.3-13.6 \log _{10} E,
$$

where $E$ is expressed in volts and $\sigma_{C E X}$ is in $\AA^{2}$.

Multiply-charged ions are neglected in CEX3D (conversely, $\mathrm{Xe}^{2+}$ ions are accounted for in both the beam and CEX ion calculations in the 2D code CEX2D [5]). While doubly-charged ions are very important for screen grid erosion (which is driven by beam ion impacts at energies near the sputtering threshold, where the yield varies steeply with energy), they have only a small effect on accelerator grid erosion rates in a thruster such as NEXT, for which doubly-charged ions make up $\lesssim 6 \%$ of the total beam ion density. Note that the cross section for the $\mathrm{Xe}^{2+}+\mathrm{Xe} \rightarrow \mathrm{Xe}+\mathrm{Xe}^{2+}$ charge 



Fig. 3 Comparison of neutral gas densities downstream of the grids calculated from the analytical model in CEX3D and a 3D Monte Carlo simulation using the DAC code.

exchange process is lower than $\sigma_{C E X}$ for singly-charged ions (Eq. 4 by a factor of $\sim 2$, and the cross section for the asymmetric process $\mathrm{Xe}^{2+}+\mathrm{Xe} \rightarrow 2 \mathrm{Xe}^{+}$is lower by an additional order of magnitude [20].

CEX3D separately calculates creation rates for CEX ions born from neutral gas flowing out of the thruster, background gas flowing toward the thruster, and background gas flowing downstream following diffuse reflections off of the grids. Each of these gas populations has a different velocity distribution, which impacts the initial velocity distribution of the CEX ions.

The calculation of flowing thruster gas density near the grids assumes that the downstream boundary of the accel grid hole acts as a disk-shaped particle sink on the upstream side and as a particle source on the downstream side [21]. The gas densities in these regions are given by:

$$
\begin{aligned}
& n_{\text {gas }}\left(z<z_{\text {accel }}\right)=n_{\text {gas, upstream }} C\left(\left(\frac{1}{C}-\frac{\delta}{2}\right)\left(1-D\left(r, z_{\text {accel }}-z, r_{\text {accel }}\right)\right)^{2}+\frac{\delta}{2}\right) \\
& n_{\text {gas }}\left(z>z_{\text {accel }}\right)=n_{\text {gas, upstream }} \frac{C h \delta}{2}\left(1+\left(\frac{1}{h}-1\right) D\left(r, z-z_{\text {accel }}, r_{\text {accel }}\right)\right),
\end{aligned}
$$

where $h$ is the ratio of the accel hole area to the beamlet area. The Clausing factor $C$ [1] and the downstream density correction factor $\delta \equiv\left\langle v_{z, \text { gas }}^{\text {upstream }}\right\rangle /\left\langle v_{z, \text { gas }}^{\text {downstream }}\right\rangle$ are determined by a Monte Carlo routine that calculates view factors for an approximate grid hole geometry and assumes diffuse reflection at grid surfaces. The function $D(r, z, a)$ is evaluated using:

$$
\begin{aligned}
\theta_{1} & =\tan ^{-1}\left(\frac{r+a}{z}\right)-\tan ^{-1}\left(\frac{r}{z}\right) \\
\theta_{2} & =-\tan ^{-1}\left(\frac{r-a}{z}\right)+\tan ^{-1}\left(\frac{r}{z}\right) \\
\cos \theta_{3} & =\sqrt{1-\frac{a^{2}-x^{2}}{r^{2}-2 r x+a^{2}+z^{2}}} \\
D(r, z, a) & =\sqrt{\left(1-\frac{1}{2}\left(\cos \theta_{1}+\cos \theta_{2}\right)\right)\left(1-\cos \theta_{3}\right)} .
\end{aligned}
$$

These formulas were carried over from previous versions of CEX2D [2] and CEX3D [3], with one correction applied (the leading Clausing factor in Eqs. 5 and 6 had been inadvertently omitted in the older code versions). Far downstream of the grids, the flowing gas density given by Eq. 6] is consistent with conservation of mass, accounting for overlap of gas flows from different grid holes.

In order to check the accuracy of the analytical gas density model, a more sophisticated 3D Monte Carlo calculation was carried out for one specific NEXT grid geometry using the DSMC Analysis Code (DAC) [22]. The results for the 
flowing neutral gas density downstream of the accel grid are shown in Fig. 3 Good agreement between the models on the order of $20 \%$ or better is evident.

The velocity distribution of the flowing gas from the thruster in CEX3D is assumed to be a drifting Maxwellian, with mean velocity obtained from the Clausing factor calculation. Based on the 3D Monte Carlo simulation results, we set the gas temperature in the downstream region to be $2 / 3$ of the discharge chamber gas temperature.

The background gas population flowing toward the grids is assumed to have a $300 \mathrm{~K}$ half-Maxwellian velocity distribution in the axial direction and $300 \mathrm{~K}$ full Maxwellian distribution in the other two directions, with density equal to half of the background neutral density in the chamber. All background gas flowing away from the thruster is assumed to have reflected off the accel grid - this gas is assumed to have a half-Maxwellian velocity distribution with the grid temperature in the axial direction, and a full Maxwellian distribution at this temperature in the other two directions. The density of the outflowing background population is chosen to make the flux of gas toward and away from the thruster equal (leakage of some incident gas through the grids into the discharge chamber is neglected). The densities of both of these background gas populations are set to zero for simulations of thruster operation in space.

If most CEX ions were born in a region with a large enough electric field to make their initial velocities inconsequential, then these precise distinctions between gas populations would not have been necessary. However, the PIC model predicts that the potential downstream of the grids can become very flat over a region spanning several centimeters (see Sec. V); thus the initial velocities play an important role in determining which CEX ions can reach the accel grid. Another important effect, which can be neglected in many other circumstances, is the finite momentum transfer in CEX collisions. Work by Araki, Patino, and Wirz has shown that approximately 55\% of CEX collisions between $1500 \mathrm{eV}$ $\mathrm{Xe}^{+}$ions and thermal Xe neutrals produce an ion with kinetic energy significantly greater than the original energy of the target atom [23-25]. Based on the experimental results and curve fits in Ref. [25], we assume in CEX3D that the distribution of CEX scattering angles is

$$
\begin{gathered}
p(\theta) \propto\left\{\begin{array}{l}
\sin \theta(\pi / 2-\theta)^{A_{C E X}}, \quad 0 \leq \theta<\theta_{C E X} \\
\sin \theta\left(\pi / 2-\theta_{C E X}\right)^{A_{C E X}}, \quad \theta_{C E X} \leq \theta \leq \pi / 2
\end{array}\right. \\
A_{C E X}=-1.0275-\left(2.35 \times 10^{-4}\right) E_{\text {beam }},
\end{gathered}
$$

where $\theta_{C E X}=1.56975 \mathrm{rad}$. Following charge exchange, the speed of the CEX ion in the atom's original rest frame is:

$$
v_{C E X}=v_{\text {beam }} \cos \theta
$$

In the limit of $90^{\circ}$ scattering, there is no momentum transferred to the CEX ion in the collision, but at smaller scattering angles, it receives a finite momentum kick. The birth velocity of the CEX ion in the thruster frame is the vector sum of $\mathbf{v}_{C E X}$ and the atom's original velocity.

Since the beam ion and neutral atom densities are assumed to be constant, the CEX ion creation rate in each computational cell only needs to be calculated once, at the initiation of the PIC simulation. Then during each PIC iteration, a CEX ion macroparticle is created at a random location within the cell with probability

$$
P=\frac{e n_{\text {beam }} V_{c e l l} \sigma_{C E X} v_{\text {beam }} n_{g a s} \Delta t}{q_{\text {min }}},
$$

where $\Delta t$ is the time step, $V_{c e l l}$ is the cell volume, and $q_{\min }$ is the minimum macroparticle charge specified in the input file. The initial macroparticle velocity is determined by randomly selecting a neutral atom velocity and CEX scattering angle from the distributions described above.

\section{B. Particle Pushing and Ion Velocity Rescaling}

CEX ion macroparticles are pushed through the beamlet using a standard "leapfrog" scheme [19] in which positions are evaluated at integer time steps while velocities are evaluated at half-integer time steps. An ion's position at time step $n+1$ is

$$
\mathbf{x}_{n+1}=\mathbf{x}_{n}+\mathbf{v}_{n+1 / 2} \Delta t=\mathbf{x}_{n}+\left(\frac{e \mathbf{E}_{n}}{M_{i}} \frac{\Delta t}{2}+\mathbf{v}_{n}\right) \Delta t
$$

The electric field at the particle location is determined by linearly interpolating from the cell boundary node potentials. Sharing of the macroparticle charge to adjacent mesh nodes after each step likewise uses a simple first-order algorithm. Interparticle collisions (except for the initial charge exchange collision) are neglected throughout the calculation. 


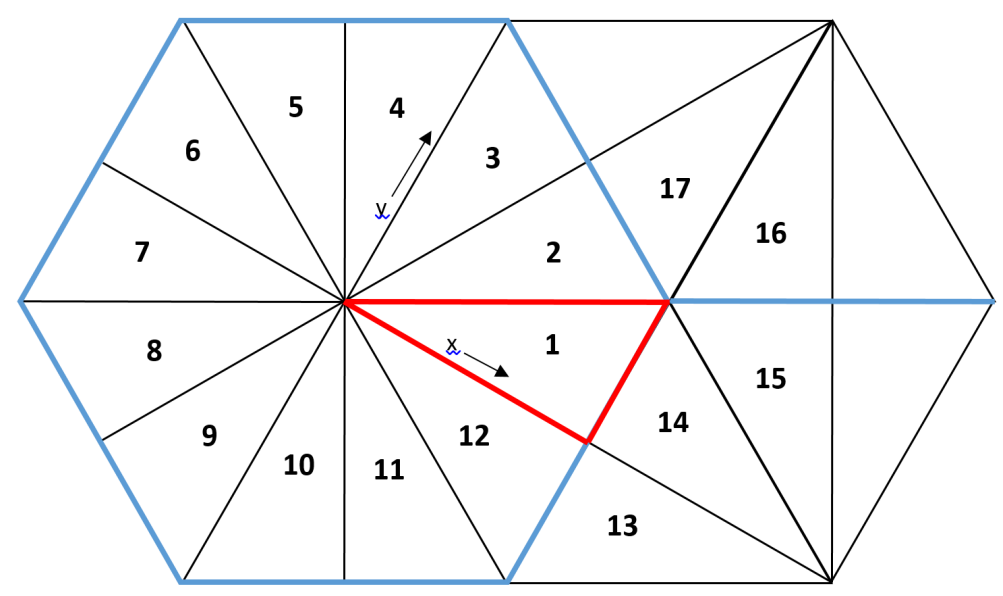

Fig. 4 Geometry for CEX ion macroparticle reflection at the domain boundaries. The figure shows a cross section of the beamlet being simulated and portions of two adjacent beamlets. The red triangle is the simulation domain, and beamlet boundaries are shown in blue.

Macroparticles that leave out the sides of the triangular domain are reflected or rotated back into the domain in a manner consistent with travel into another section of the beamlet or into an adjacent beamlet, given the hexagonal symmetry. This situation is illustrated in Fig. 4. A macroparticle starting within the simulation domain (Triangle \#1, outlined in red) can end up in any of the other 16 numbered triangles after a single time step that satisfies the CFL condition (discussed below). The transformations applied to the particle's position and velocity in order to put it back into Triangle \#1 are, for each of the possible destination regions:

- Triangle \#3, \#5, \#7, \#9, or \#11: Rotate the position and velocity vectors clockwise by $60,120,180,240$, or 300 degrees, respectively.

- Triangle \#2, \#4, \#6, \#8, \#10, or \#12: Rotate the position and velocity vectors clockwise by 60, 120, 180, 240, 300, or 0 degrees, respectively. Then reflect the position and velocity over the $x$-axis.

- Triangle \#13: Translate the origin to the center of the lower right beamlet in the figure $\left(x \rightarrow x-2 x_{\text {max }}\right)$. Then rotate the position and velocity vectors by 180 degrees.

- Triangle \#14: Reflect the position and velocity vectors over the line $x=x_{\text {max }}$.

- Triangle \#15: Translate the origin to the center of the lower right beamlet. Then rotate the position and velocity vectors clockwise by 120 degrees.

- Triangle \#16: Translate the origin to the center of the upper right beamlet $\left(x \rightarrow x-x_{\max } ; y \rightarrow y-\right.$ $\left(y_{\max }+\sqrt{x_{\text {max }}^{2}+y_{\text {max }}^{2}}\right)$. Rotate the position and velocity vectors clockwise by 300 degrees. Then reflect the position and velocity vectors over the $x$-axis.

- Triangle \#17: Translate the origin to the center of the upper right beamlet. Then rotate the position and velocity vectors clockwise by 240 degrees.

Here $x_{\max }$ and $y_{\max }$ are the lengths of the simulation domain along the two directions (i.e., the lengths of the legs of the triangle). Note that this calculation implicitly assumes that the dynamics in the adjacent beamlet (and in all other beamlets in the thruster) mirrors that in the beamlet being simulated. The boundary conditions used for the potential solver rely on the same approximation, which neglects variations in the beam current density with radial position.

A general requirement for stable PIC simulations is that the time step should be short enough that particles cross no more than one mesh volume in a single time step (this is known as the Courant-Friedrichs-Lewy or CFL condition - actually, the requirements in two and three dimensions are slightly more stringent than $v \Delta t<\Delta x$ ). This condition poses a significant challenge for ion thruster PIC simulations because of the wide range of CEX ion velocities present near the grids. CEX ions born near the screen grid may be accelerated to nearly the full beam energy, which for $V_{b}=2 \mathrm{kV}$ corresponds to $v \approx 50 \mathrm{~km} / \mathrm{s}$ for xenon. A typical Debye length near the screen grid is $5 \times 10^{-5} \mathrm{~m}$, so the mesh spacing in this region should be no larger than $\Delta x \approx 3 \times 10^{-5} \mathrm{~m}$. The CFL condition then limits the time step to be $\Delta t \leq 0.6 \mathrm{~ns}$. However, CEX ions downstream of the grids have energies on the order of $0.1 \mathrm{eV}(v \approx 400 \mathrm{~m} / \mathrm{s})$, and the simulation needs to be able to follow these ions over distances of several $\mathrm{cm}$ and allow multiple transit times for the 


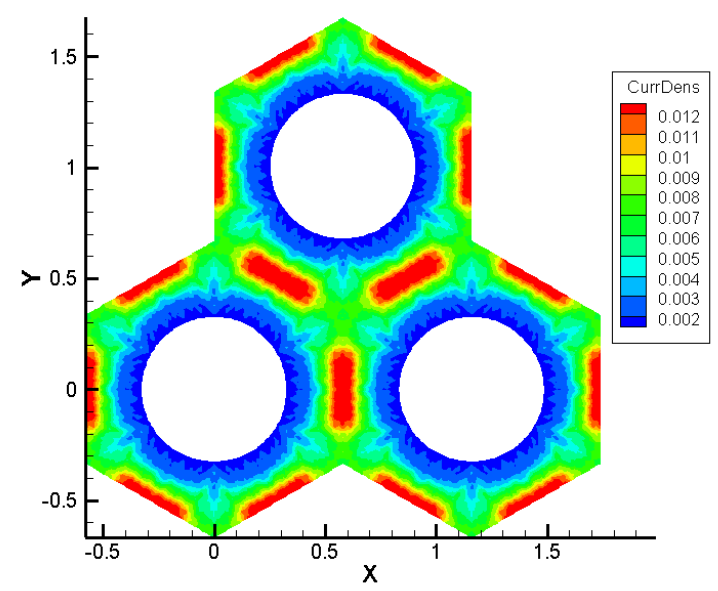

(a)



(b)

Fig. 5 Current density incident on the downstream face of the accel grid for the maximum beamlet current at the $1800 \mathrm{~V}, 1.2$ A NEXT operating condition (TL12) under space vacuum, with velocity rescaling threshold set to $v_{\max }=8000 \mathrm{~m} / \mathrm{s}(\mathbf{a})$ or $v_{\max }=1000 \mathrm{~m} / \mathrm{s}(\mathbf{b})$.

calculation to reach a steady state. The simulation duration thus needs to be on the order of $1 \mathrm{~ms}$, corresponding to $>10^{6}$ timesteps. Given that each step can take on the order of a minute of computation time for relatively high beamlet current operating condition (such as NEXT TL40), these considerations seem to render 3D simulations completely impractical on a personal computer.

We have overcome this obstacle by adopting a rescaling procedure to limit CEX ion macroparticle velocities to a low value on the order of $1-2 \mathrm{~km} / \mathrm{s}$ (adjustable in the CEX3D input file), so that longer time steps can be taken. The algorithm relies on the fact that particles of different charge-to-mass ratios starting from rest follow the same trajectories in a steady state electrostatic field, although they move with different velocities [5]. Thus as long as a CEX ion's birth velocity is small compared to the threshold for rescaling, we can adjust the magnitude of the velocity as it moves without changing the steady state potential solution or the CEX ion current incident on the grids. The macroparticle's charge and mass must also be adjusted to maintain the same space charge density and ensure that the velocity magnitude is consistent with the value of $q / m$ so that the particle stays on the same trajectory. Specifically, since the kinetic energy $K$ after acceleration through a potential $V$ is proportional to $q$, the velocity scales as $v \sim \sqrt{K / m} \sim \sqrt{q / m}$. Thus if we multiply $v$ by a factor $\gamma, q / m$ must be multiplied by a factor $\gamma^{2}$. The local space charge density is $\rho \sim n q \sim q / v$, so to maintain the same space charge density after velocity rescaling by $\gamma, q$ must also be multiplied by $\gamma$. Thus $m$ must be multiplied by $1 / \gamma$.

After each particle pushing step, CEX3D checks if the macroparticle's speed exceeds the velocity rescaling threshold $v_{\max }$; if it does, rescaling is carried out with $\gamma=v_{\max } / \sqrt{v_{x}^{2}+v_{y}^{2}+v_{z}^{2}}$. The charge rescaling is carried out in two steps, with $q$ multiplied by $\gamma_{1}=(1+\gamma) / 2$ prior to sharing the macroparticle's charge to adjacent mesh nodes and then by $\gamma_{2}=2 \gamma /(1+\gamma)$ afterwards, for a total charge rescaling $\gamma_{1} \gamma_{2} \equiv \gamma$. This procedure sets the charge shared to the mesh to a value halfway between the macroparticle's old and new charges; since it spent the previous time step moving at the old velocity and will spend the next time step moving at the new velocity, distortion of the local space charge density is avoided to first order.

When the simulation reaches a steady state, the CEX ion current to the grids is determined entirely by the birth rate of CEX ions at positions from which the electric field pushes ions back to the grids, and we can calculate the current density to surfaces by simply adding up the total macroparticle charge collected per unit time, provided we use each particle's original charge at birth (rather than its rescaled charge). Equivalently, since current density is $J=n q v$ and $n \sim 1 / v$, the current density scales as $J \sim q$, which implies that velocity rescaling does not affecting the grid currents calculated from the macroparticle's original charge. The sputter yield produced by a CEX ion incident on a grid must be calculated using the ion's physical kinetic energy (rather than the rescaled value), which is readily determined from the potential difference between the ion's birth location and the grid. 


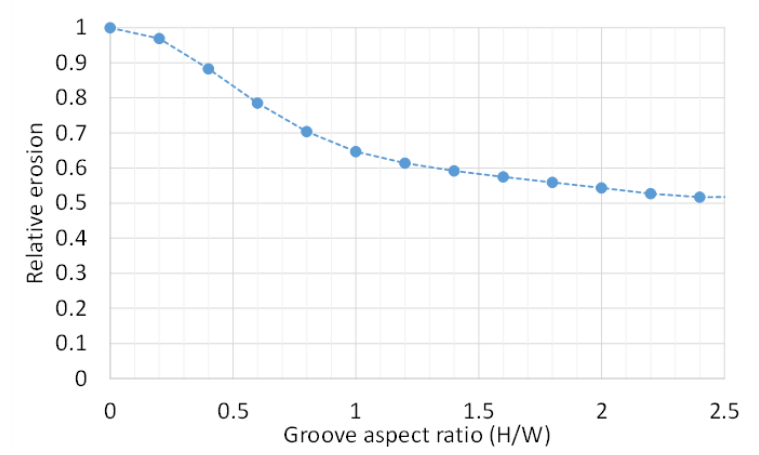

(a)

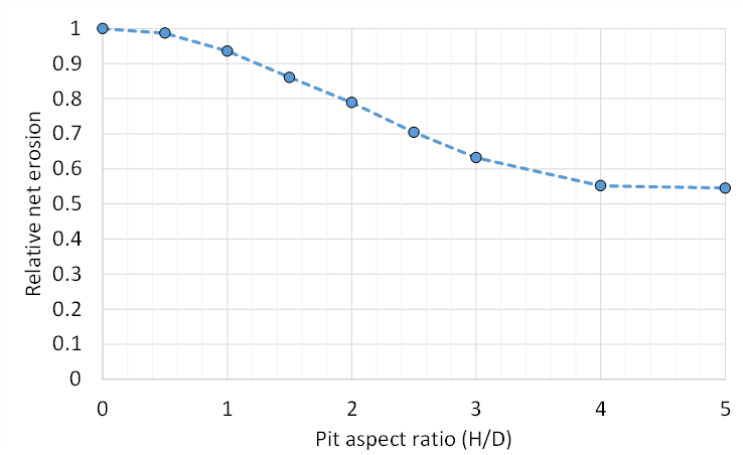

(b)

Fig. 6 Decrease in net erosion rate at the bottom of grooves (a) and pits (b) resulting from re-deposition of sputtered material, calculated using the COLISEUM code [26].

The time step is calculated prior to each particle pushing step so that all macroparticles in the domain satisfy $v_{x} \Delta t \leq f \Delta x$ (and likewise for the $y$ and $z$ velocity components), where $f$ is an adjustable multiplier on the CFL limit. In practice, using $f=2$ causes no significant problems for CEX3D simulations, and at somewhat higher values the main issue is a breakdown of the boundary rotation/reflection algorithm described above, which could likely be overcome with modifications to this algorithm. Satisfying the CFL condition is not strictly necessary because CEX ions are usually only accelerated to high velocities near the accel grid or upstream of it, and in these regions their space charge has little influence on the potential structure.

In order to reduce the computation time, the CEX ion calculation begins with several thousand iterations in which CEX ions are created and pushed through the static potential calculated by the beam ion module. Once the number of macroparticles in the domain has built up to near its steady state value, the code begins to account for the CEX ion space charge and update the potential after every time step. Charge incident on the grids and mass loss rates are recorded for a subset of these PIC iterations at the end of the simulation, which should be chosen to correspond to a time when the calculation has approximately reached a steady state. The code writes a restart file that can be used to resume simulations from the latest point in the PIC calculation.

The CEX ion velocity/charge/mass rescaling approach was validated by comparing simulations results for the NEXT $1800 \mathrm{~V}, 1.2 \mathrm{~A}$ throttle level (TL12) obtained with velocity rescaling thresholds set to $1000 \mathrm{~m} / \mathrm{s}$ and $8000 \mathrm{~m} / \mathrm{s}$. For the maximum beamlet current at this operating condition, the accel grid current (normalized to the beamlet current) was $I_{a} / I_{b}=2.88 \times 10^{-4}$ for the $v_{\max }=1000 \mathrm{~m} / \mathrm{s}$ simulation and $I_{a} / I_{b}=3.00 \times 10^{-4}$ for the $v_{\max }=8000 \mathrm{~m} / \mathrm{s} \mathrm{simulation}$. The current density distributions on the downstream face of the accel grid are compared in Fig. 5 . The main features of the erosion pattern are similar between the two cases, but it appears that a lower fraction of CEX ions are funneled into the deep groove pattern in the case with aggressive velocity rescaling.

The modest discrepancy between the two cases may be attributed in part to the fact that these simulations were run for $\sim 345 \mu \mathrm{s}$, which was not long enough to completely reach steady state. When the $v_{\max }=1000 \mathrm{~m} / \mathrm{s}$ simulation was continued out to $1.05 \mathrm{~ms}$, the accel grid current increased by $42 \%$ (continuing the $v_{\max }=8000 \mathrm{~m} / \mathrm{s}$ simulation for a similar duration was not practical due to the factor of 8 longer computation time). It also must be noted that some CEX ions in the simulations are born with velocities on the order of $1000 \mathrm{~m} / \mathrm{s}$ - since the validity of the rescaling procedure relies on the assumption that the ions have negligible initial velocity, some error in the calculated trajectories of these macroparticles is to be expected. Nevertheless, the benefits of velocity rescaling in terms of computational efficiency outweigh the small loss in accuracy.

\section{Erosion Rates and Removal of Particles from the Computation}

After every particle pushing step, the code checks if the macroparticle has exited the domain or reached a grid, and if so it removes them from the simulation. During the final set of iterations over which erosion and grid currents are calculated, detailed information about each CEX ion that hit a grid (such as macroparticle charge, birth and death positions, impact energy, and impact angle) are written to the output file. Grid sputtering rates are calculated using the 
best fit energy- and angle-dependent sputter yields for xenon impacting molybdenum and carbon from the literature survey in Ref. [27]. The yields can easily be adjusted to other values. For ion impact energies on the order of $200 \mathrm{eV}$, better agreement between simulated and measured erosion rates has been achieved when using the lower $50 \%$ confidence bound for the moly sputter yield from Ref. [27] rather than the maximum likelihood value.

At present, CEX3D does not account for re-deposition of sputtered atoms within the pits and grooves on the accel grid downstream face. On the other hand, the two-dimensional code CEX2D does account for an average re-deposition rate on the accel grid hole walls. Stand-alone modeling of pits and grooves re-deposition was carried out using the COLISEUM code [26] to determine the extent to which CEX3D would be expected to overestimate the net erosion rate during the NEXT LDT. The CEX3D-generated accel grid current density profiles were used as the ion source term in COLISEUM which then assessed sputtering and redeposition of the grid material for sample pit and groove geometries of varying depths and corresponding aspect ratios. The differential sputtering model was based on the modified-Zhang profiles as measured and estimated in Ref. [28]. Ratios of the relative erosion rate at the bottom of the pit or groove with and without redeposition effects were calculated and are plotted as a function of the respective aspect ratios in Fig. 6 .

As discussed above, reflection of CEX ions when they reach the $x=x_{\max }$ boundary of the domain is used to simulate ions leaving the beamlet and being replaced by ions entering from adjacent equivalent beamlets. Since the beam has finite extent, there is a limit to how many times these reflections can occur. The code incorporates this global effect in an approximate way by tracking the total cumulative displacement of each macroparticle in the $x y$-plane and killing the macroparticle once this displacement exceeds the beam radius. Phyically, macroparticles entering the grid-center beamlet cannot have traveled so far radially that they would have been born outside the beam.

\section{Potential Solver}

Electrons in the PIC CEX ion calculation are modeled as a fluid that obeys the Boltzmann relation, as in the beam ion calculation, and Poisson's equation is linearized using Eq.11. Along the downstream domain boundary, the potential at one point (where the beam ion density is maximized) is set to a fixed reference value, and the Boltzmann relation is used to calculate the potential elsewhere on the boundary, using the total beam plus CEX ion density from the previous PIC time step.

In thrusters with domed (convex) grids, beam divergence causes the beam ion density to decrease moving away from the grids. In the quasineutral plume plasma, the Boltzmann relation implies that there should be an axial potential gradient satisfying $\phi(x)=\phi_{0}+\left(k_{B} T_{e} / e\right) \ln \left(n_{e} / n_{e 0}\right)$. The potential gradient arising from beam divergence plays an important role in limiting the collection volume from which CEX ions can flow back to the accel grid. This effect is approximately accounted for within CEX3D's potential solver by multiplying the beam, CEX ion, and electron densities

at a distance $z$ downstream of the screen grid by a factor $R_{\text {optics }}^{2} /\left(z+R_{\text {optics }}\right)^{2}$, where $R_{\text {optics }}$ is the grid radius of curvature. This correction factor does not fully account for the decreasing neutral gas density moving away from the grids, which affects the local CEX ion creation rates-this feature will be incorporated in a future code version.

\section{Results and Comparisons with NEXT LDT Data}

Fig. 7 7 shows an example of the calculated potential profile downstream of the grids for the NEXT $1800 \mathrm{~V}, 1.2 \mathrm{~A}$ throttle point (TL12). When beam ions alone are considered, the potential closely follows the Boltzmann relation. However, when CEX ions are added to the calculation, their density builds up preferentially in the downstream region (see Fig. 8), flattening the potential. This result seems surprising, but the limited data presently available for NEXT (for example, see Fig. 11 in Ref. [29]) suggests that the axial potential gradient in the first $45 \mathrm{~cm}$ downstream of the grids is indeed significantly flatter than we would expect from considerations of the beam density and Boltzmann relation alone.

A simple 1D analytical calculation is helpful for understanding why the CEX ion density increases moving away from the grids in the simulations, even though ions born from a planar source at a single axial location would have their density decrease as they accelerated down the potential hill set up by the beam ions. Consider a situation with a uniform axial electric field $E$, so that the potential satisfies $\phi(z)=-E z$. Assume all CEX ions are born flowing downstream with velocity $v_{0}$. Then for ions born at position $z_{b}<z<\left(z_{b}+d z_{b}\right)$, the velocity satisfies

$$
v\left(z ; z_{b}\right)=\sqrt{v_{0}^{2}+\frac{2 e E\left(z-z_{b}\right)}{M_{i}}} .
$$




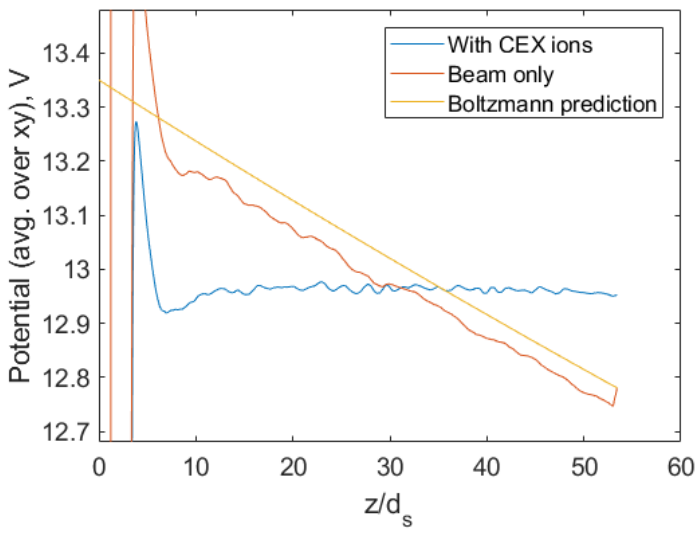

Fig. 7 Downstream plasma potential averaged over the $x y$-plane for the maximum beamlet current at the 1800 V, 1.2 A NEXT operating condition (TL12) under space vacuum.

If these ions are born at a steady rate $j_{b}$ per unit volume, then their density at position $z$ is

$$
n\left(z ; z_{b}\right)=\frac{j_{b} d z_{b}}{v\left(z ; z_{b}\right)}=j_{b} d z_{b}\left(v_{0}^{2}+\frac{2 e E\left(z-z_{b}\right)}{M_{i}}\right)^{-1 / 2} .
$$

To find the total CEX ion density including contributions from ions born at all locations, integrate this expression from $z_{b}=0$ to $z_{b}=z$ :

$$
n(z)=\int_{0}^{z} j_{b} d z_{b}\left(v_{0}^{2}+\frac{2 e E\left(z-z_{b}\right)}{M_{i}}\right)^{-1 / 2}=\frac{j_{b} M_{i} v_{0}}{e E}\left(\sqrt{1+\frac{2 e E z}{M_{i} v_{0}^{2}}}-1\right),
$$

which shows that the density is an increasing function of $z$, regardless of the relative magnitudes of $v_{0}$ and $E$. Here we have considered only CEX ions born from flowing gas, but in fact the presence of background gas with near zero mean velocity exacerbates this effect.

In a real thruster, the tendency for the CEX ion density to build up at downstream positions is offset by radial losses out of the beam. This underscores the importance of accounting for these losses in a single-beamlet code like CEX3D, and also shows that a precise treatment of the initial CEX ion velocity distribution (see Sec. IV.A) is critical for accurately predicting the CEX ion current incident on the accel grid.

Simulated downstream potential contours for a 2D slice of the beamlet at the NEXT 1800 V, 3.52 A full-power operating point (TL40) are shown in Fig. 9 The figure compares the potential before and after accounting for CEX ion space charge, illustrating how the CEX ions act to smooth out potential nonuniformities set up by density variations in the beam.

For CEX3D simulations of the NEXT LDT, the relatively flat calculated potential downstream of the grids meant that domain lengths on the order of $50 d_{s}$ were necessary in order to fully encompass the source region for CEX ions that contribute to accel grid sputtering. The collection volume was particularly large in simulations of ground tests with finite background pressure, since there is a component of the background gas flowing toward the thruster (in contrast, the mean downstream velocity of the thruster gas causes CEX ions born from this population to stream away from the grids when the potential is flat). Fig. 10 shows the creation rates of CEX ions that go on to sputter the accel grid hole wall or downstream face, for the $1179 \mathrm{~V}, 3.52$ A NEXT operating condition (TL37). The contributions to pits and grooves erosion by CEX ions born from thruster gas and background gas are shown separately.

During the NEXT LDT, the thruster was run at 5 throttle levels that were roughly located at the corners of the throttle table in $I_{b}-V_{b}$ space [15, 30] and thus were expected to bound the possible range of grid erosion phenomena. CEX3D simulations were carried out for each of these throttle points with background gas pressures ranging from space vacuum to 4 times the nominal LDT facility pressure. Simulated erosion patterns on the accel grid downstream face for the flight cases are shown in Fig. 11. Generally, groove erosion dominates over pit erosion for low perveance (high $V_{b}$ and/or low $I_{b}$ ) conditions, whereas pit erosion dominates for high perveance conditions. This is consistent with the measured trend from the LDT toward greater groove-to-pit depth ratio at larger radii, and also consistent with our observation that 


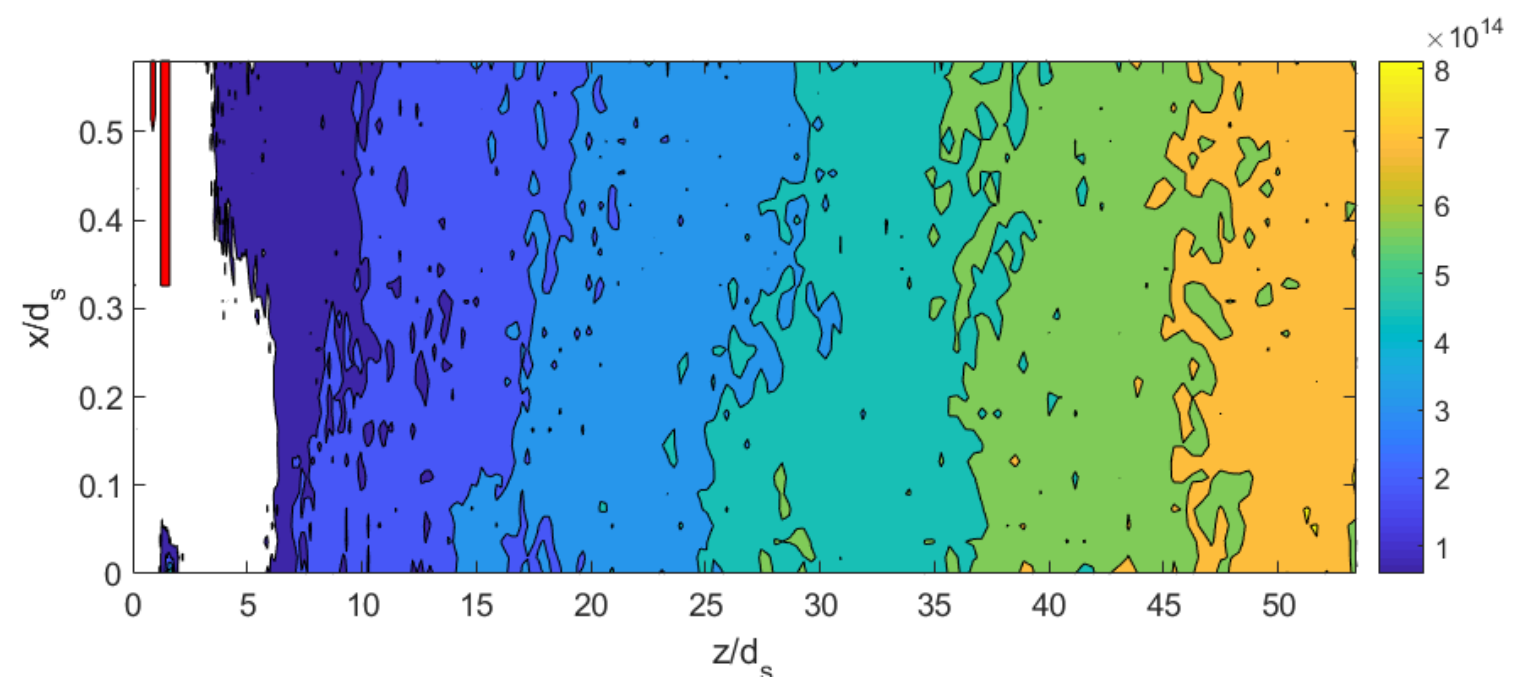

Fig. 8 CEX ion density contours in the $x z$-plane for the maximum beamlet current at the $1800 \mathrm{~V}, 1.2 \mathrm{~A}$ NEXT operating condition (TL12) under space vacuum. Note that the aspect ratio of this plot and those that follow is stretched in the $x$-direction for readability.

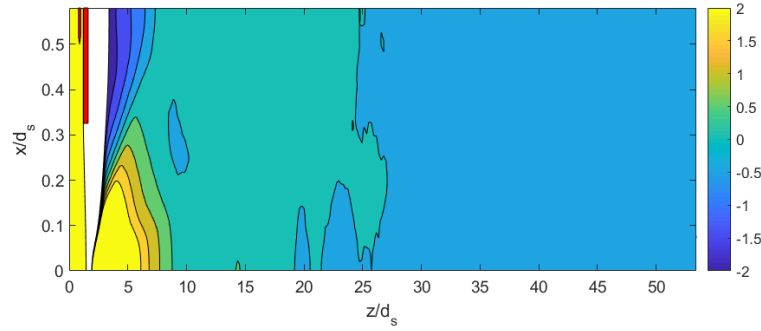

(a)



(b)

Fig. 9 Potential contours, relative to the downstream boundary reference potential, for the maximum beamlet current at the $1800 \mathrm{~V}, 3.52$ A NEXT operating condition (TL40) with the nominal LDT background pressure. a) Potential produced by beam ions alone. b) Potential including both beam ion and CEX ion space charge.

increasing the grid gap (which moves a given throttle level closer to the perveance limit [1]) in simulations of NEXT TL40 caused the dominant erosion feature to transition from grooves to pits. The simulations predict a unique erosion pattern for NEXT TL1, which has high perveance and a very low ratio $R \equiv V_{b} /\left(V_{b}+V_{a c c e l}\right)<0.5$.

In Fig. 12 model predictions for the accel grid current as a function of background pressure are compared with data taken near the end of the LDT. The finite pressure simulations were carried out with a customized accel grid geometry that approximated the grid shape with accumulated carbon deposits at the end of the LDT (the details of the eroded geometry of the accel grid downstream face, which have a much smaller effect on beam ion and CEX ion trajectories than the overall thickening of the accel grid due to carbon deposition, were not accounted for). On the other hand, the zero pressure (space) simulations used the nominal NEXT grid geometry. The accel grid current for each of these zero pressure simulations fell close to a linear fit extrapolated from the finite pressure simulations, suggesting that the $I_{a} / I_{b}$ vs. $P$ scaling was similar for the beginning-of-life and end-of-LDT grid geometries. Comparing the model results for space with an extrapolation of the data to zero pressure, it appears that the simulations overestimate the accel grid current at the high current operating points (TL37, TL40) and underestimate the current at the low current operating points (TL1, TL5, TL12). The slope of the pressure dependence is overestimated in all cases. This implies that the simulations were overestimating the contribution to the accel grid current from CEX ions born from background gas. The underlying source of this error is probably inaccuracy in the downstream region potential solution or neglect of a 


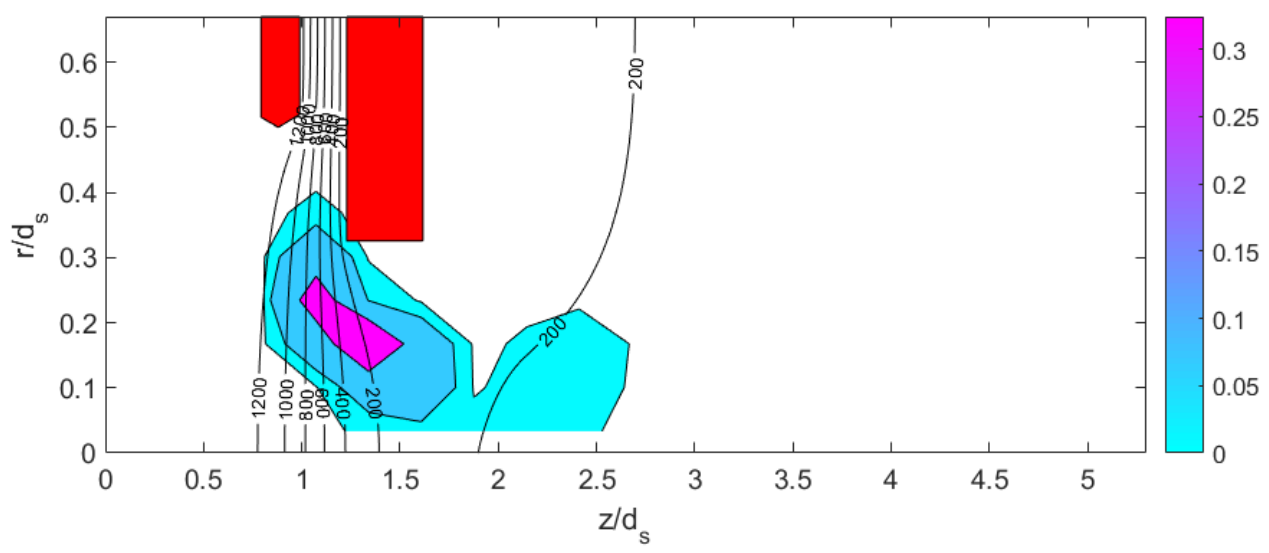

(a)

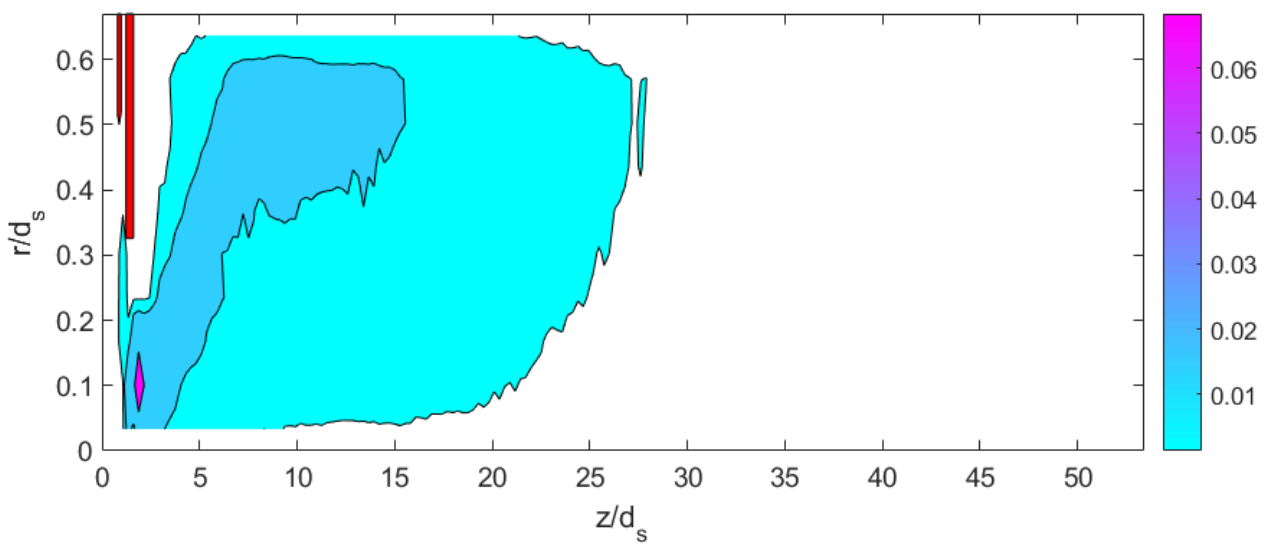

(b)

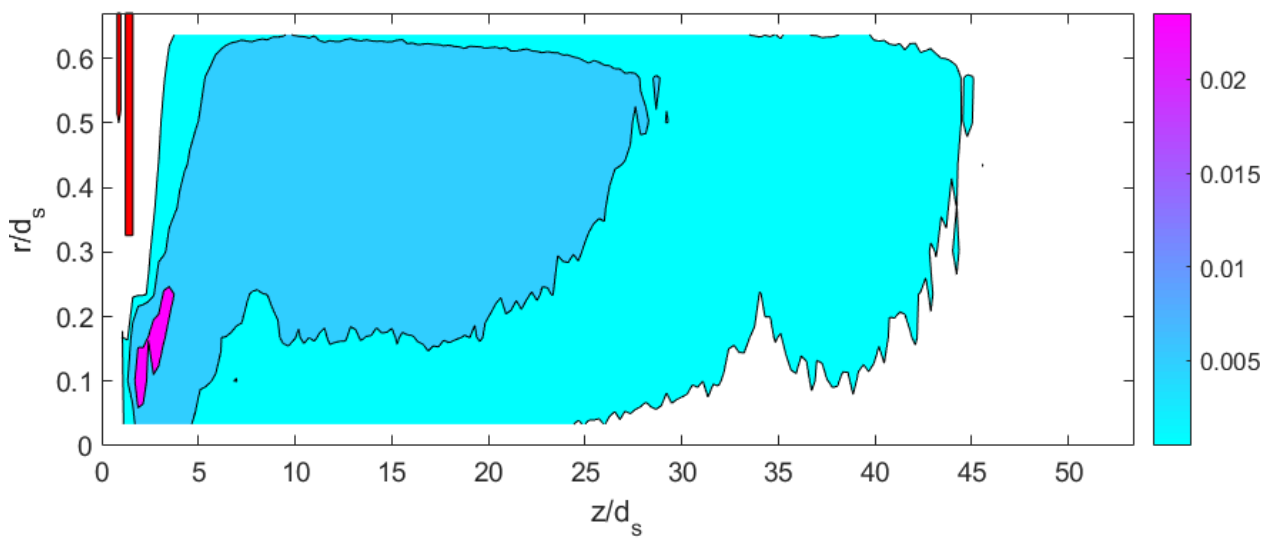

(c)

Fig. 10 Birth locations of CEX ions causing accel grid erosion for the maximum beamlet current at the 1179 V, 3.52 A NEXT operating condition (TL37) with the nominal LDT background pressure. The creation rates are integrated over the azimuthal direction and plotted as a function of $r$ and $z$ with units of $\mathrm{A} / \mathrm{m}^{2}$. a) Total creation rate for CEX ions that cause barrel erosion. Contour lines show the plasma potential with respect to the accel grid. b) Creation rate for pits and grooves CEX ions born from the thruster gas flow. c) Creation rate for pits and grooves CEX ions born from background gas. Note that the colorbar scales are different in each of the three plots. 


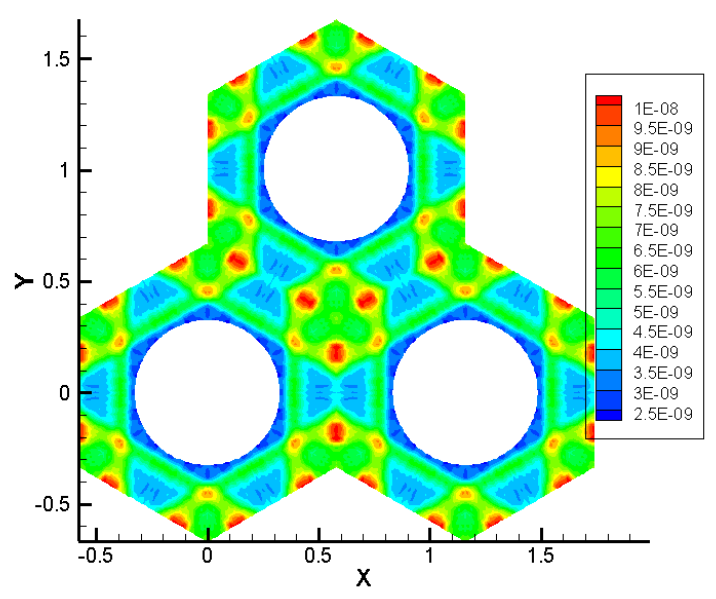

(a)

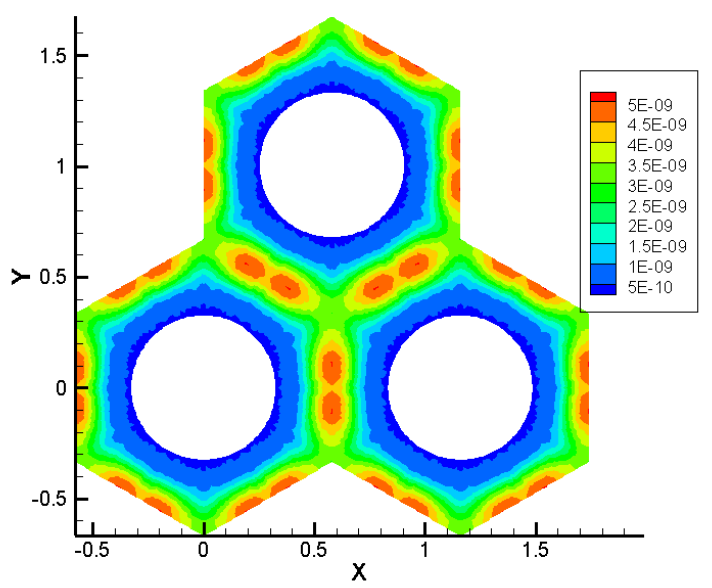

(c)



(b)



(d)

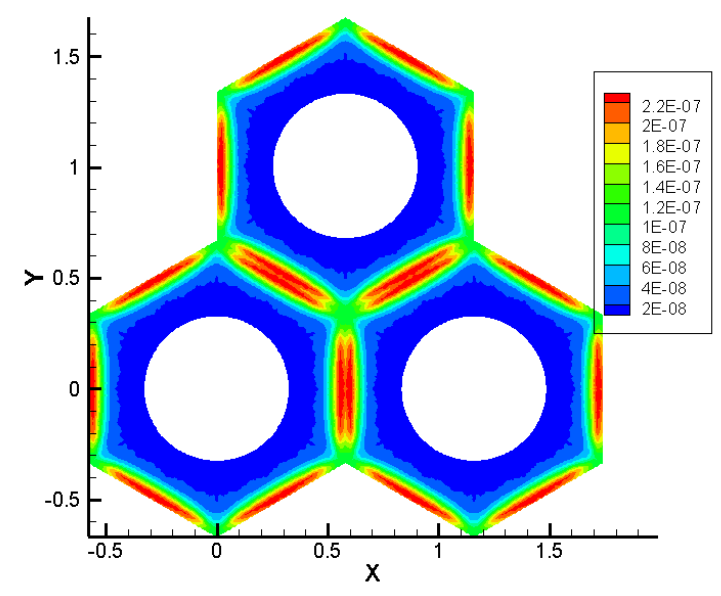

(e)

Fig. 11 Simulated erosion patterns on the accel grid downstream face for the maximum beamlet current at the 5 NEXT operating conditions studied, under space vacuum. a) 275 V, 1.0 A (TL1). b) 679 V, 1.2 A (TL5). c) $1800 \mathrm{~V}, 1.2 \mathrm{~A}$ (TL12). d) $1179 \mathrm{~V}, 3.52$ A (TL37). e) $1800 \mathrm{~V}, 3.52$ A (TL40). 


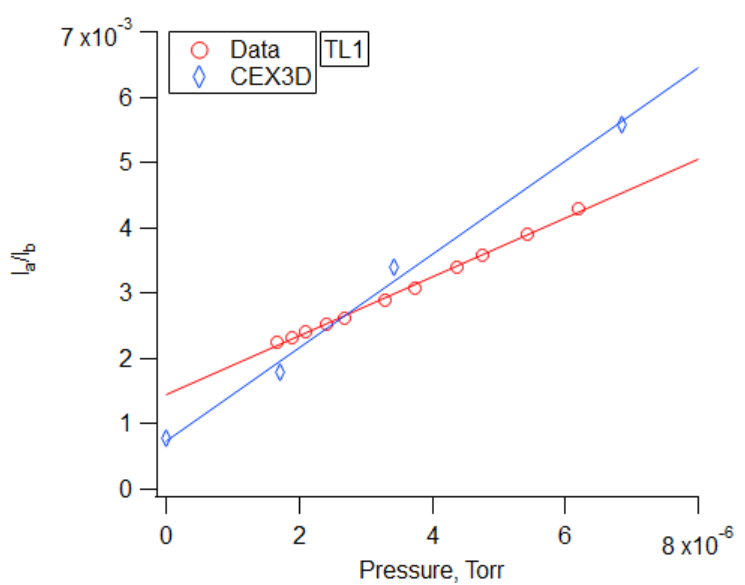

(a)

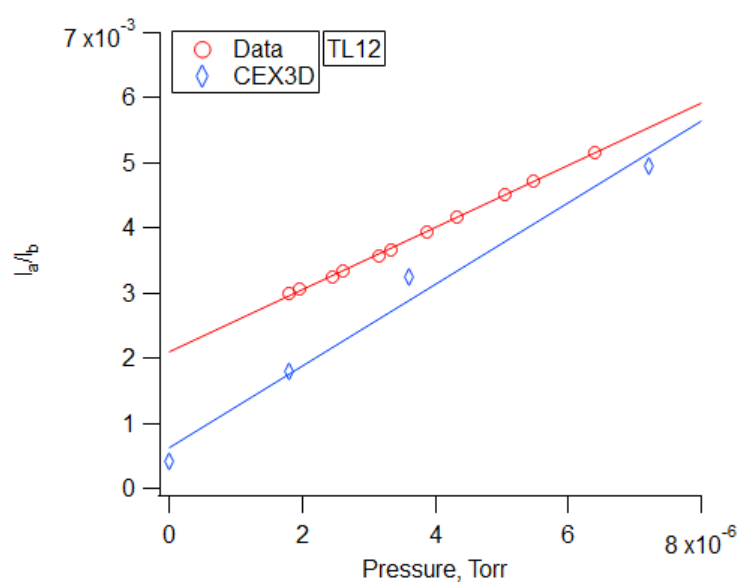

(c)

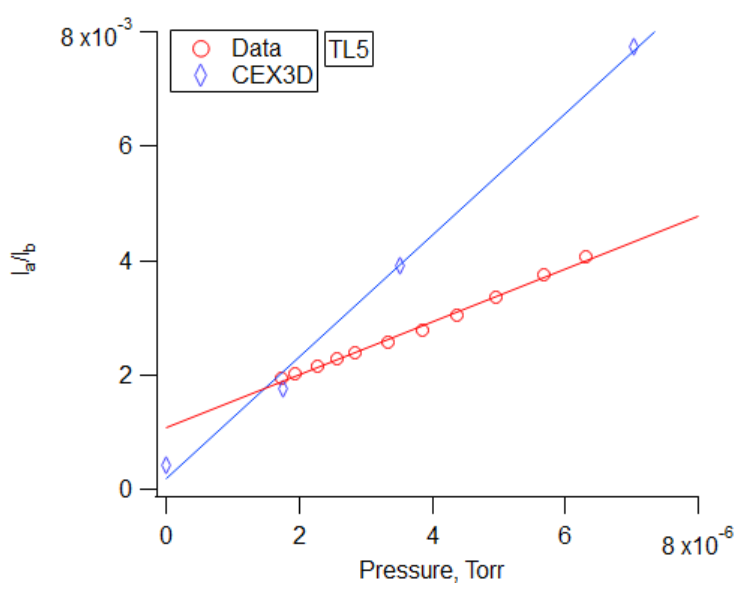

(b)



(d)



(e)

Fig. 12 Measured and simulation ratio of accel grid current to beamlet current for the 5 NEXT operating conditions studied. In plotting the data, a factor of 2.5 gas species correction was applied to the pressures measured by an ion gauge calibrated on nitrogen. The simulations results shown are for the maximum beamlet current at each throttle point. Linear fits to the data and model results are shown. a) 275 V, 1.0 A (TL1). b) 679 V, $1.2 \mathrm{~A}$ (TL5). c) $1800 \mathrm{~V}, 1.2 \mathrm{~A}$ (TL12). d) $1179 \mathrm{~V}, 3.52$ A (TL37). e) $1800 \mathrm{~V}, 3.52 \mathrm{~A}$ (TL40). 
Table 1 Breakdown of sources of accel grid current for the maximum beamlet current at the 5 NEXT operating conditions modeled, for the nominal LDT background pressure. Currents are listed in nA for a single beamlet.

\begin{tabular}{l|c|cc|cc}
\hline Throttle Level & Total Current & \multicolumn{2}{|c|}{ Barrel Current } & \multicolumn{2}{c}{ Pits and Grooves Current } \\
& & Thruster Gas & Background Gas & Thruster Gas & Background Gas \\
\hline TL1 (275 V, 1.0 A) & 128 & 24 & 4 & 44 & 56 \\
TL5 (679 V, 1.2 A) & 121 & 13 & 3 & 32 & 72 \\
TL12 (1800 V, 1.2 A) & 134 & 3 & 1 & 43 & 87 \\
TL37 (1179 V, 3.52 A) & 1139 & 134 & 16 & 515 & 474 \\
TL40 (1800 V, 3.52 A) & 1434 & 70 & 11 & 720 & 634 \\
\hline
\end{tabular}

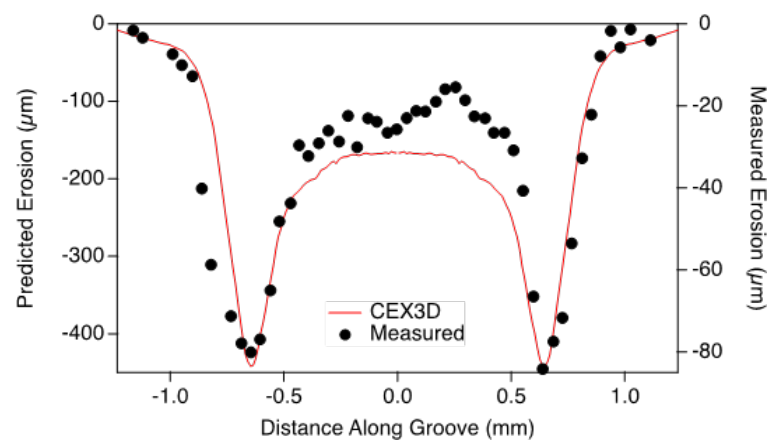

Fig. 13 Eroded depth along a groove at the grid center for the NEXT 2000 hour test $(1800 \mathrm{~V}, 3.52 \mathrm{~A}$, TL40).

global radial electric field that acts to sweep CEX ions out of the beam.

It should be noted that there was experimental evidence for direct impingement of beam ions on the accel grid during operation at TL12 during the LDT. A non-zero beam ion contribution to the accel grid current was also predicted by CEX3D simulations of a beamlet with $60 \%$ of the maximum current for the operating point (representing a beamlet located near the radial edge of the grid hole pattern). This may explain the large discrepancy between the measured $I_{a} / I_{b}$ ratio and the simulation result for the maximum current beamlet at this operating point.

Simulations using $60 \%$ of the peak beamlet current at the other LDT operating points (with the nominal LDT background pressure) produced $I_{a} / I_{b}$ that agreed with the ratio for the maximum current beamlet to within $12 \%$, except for at TL40, where the ratio differed by $27 \%$. This result implies that minimal error was introduced by treating the $I_{a} / I_{b}$ ratio modeled for the maximum current beamlet alone as a proxy for the overall ratio across the whole beam. This is particularly reasonable given that NEXT has a relatively flat current density profile across a large fraction of the beam [15].

Table 1 presents a detailed breakdown of the sources of accel grid current in the CEX3D simulations. At all 5 NEXT operating points, the majority of the CEX ion current incident on the accel grid struck the downstream face. At the nominal LDT background pressure, the current incident on the hole walls primarily consisted of CEX ions born from thruster gas. At TL1, TL37, and TL40, the thruster gas and background gas made similar contributions to the pits and grooves current, while at TL5 and TL12, the background gas contribution was larger by a factor of $\sim 2$.

Since interpretation of the erosion patterns from the LDT was complicated by facility carbon deposition, the CEX3D erosion calculations were validated against the results from the NEXT 2000 Hour Test [31], which was carried out at the $1800 \mathrm{~V}, 3.52 \mathrm{~A}$ operating condition (TL40). The molybdenum sputter yield as a function of energy used in the simulations corresponded to the lower bound of the 50\% confidence interval from Ref. [27]. A comparison of modeled and measured eroded depths is shown in Fig. 13. Unlike for TL40 operation during the LDT (see Fig. 11(e) and Ref. [15]), the pits were eroded faster than the grooves during the 2000 Hour Test (likely due to a difference in the hot grid gap). CEX3D was able to predict the erosion profile fairly accurately, but the magnitude of the erosion rate was over-predicted by a factor of $\sim 5$ in the pits and $6-8$ in the grooves.

Part of this discrepancy can be attributed to the factor of $\sim 2$ overprediction of the accel grid current at TL40 (Fig. 12(e) . However, it is difficult to explain the remaining factor of 2-4, given that ions causing pits and grooves 
erosion strike the grid at nearly normal incidence and have minimal energy dispersion, so there should be a simple proportionality between incident current density and erosion rate. The pits and grooves were relatively shallow after 2000 hours of testing (aspect ratio < 1:1), implying that re-deposition of sputtered material would not have been expected to reduce the net erosion rate by more than $20 \%$ (see Fig. 6). One possibility is that a large fraction of the measured accel grid current was collected at radii beyond the edge of the hole pattern. It is also possible, but unlikely, that there is some unidentified thruster ground test effect that reduces the sputter yield on the accel grid below the values determined from dedicated short-duration sputter yield measurements. It is interesting to note that a similar discrepancy between grid current and erosion rate was identified 20 years ago during NASA Solar Application Readiness (NSTAR) life testing [32].

\section{Conclusion}

We have presented upgrades to NASA's CEX3D code for improved simulations of accelerator grid life in two-grid ion thrusters. Using a PIC calculation for CEX ions, the pits and grooves erosion pattern on the accel grid downstream face is calculated, accounting for CEX ion space charge that modifies the potential downstream of the grids and alters the collection volume from which CEX ions can flow back to the grid. The code also calculates accel grid hole wall (barrel) erosion, which is mostly caused by CEX ions born near the grids, and it is capable of simulating three-grid ion optics as well. With no variable inputs or free parameters, the code was able to predict accel grid currents for NASA's NEXT thruster that agreed with results from the Long Duration Test (LDT) to within a factor of $\sim 2$. To achieve better agreement than this, it is likely that the single-beamlet calculations presented here will need to be joined with a global beam (plume) model in order more accurately account for the real variation in beamlet currents across the beam, which affects the boundary conditions in the beamlet simulations and creates a global radial electric field that can push CEX ions out of the beam.

The eroded depth in the pits and grooves in simulations of the NEXT full-power operating condition was overpredicted by a larger factor than the accel grid current. In the future, a detailed model for re-deposition of eroded atoms within the pits and grooves pattern will be incorporated in CEX3D. However, even with this change it appears that a significant inconsistency between the grid current and downstream erosion will remain - this discrepancy is readily evident in simple hand calculations, and it has also been observed for the NASA's NSTAR thruster in both earlier papers and recent simulations. Clearly more investigation is warranted to understand this phenomenon.

\section{Acknowledgments}

This research was carried out at the Jet Propulsion Laboratory, California Institute of Technology, under a contract with the National Aeronautics and Space Administration.

\section{References}

[1] Goebel, D. M., and Katz, I., Fundamentals of Electric Propulsion: Ion and Hall Thrusters, John Wiley \& Sons, Inc., Hoboken, NJ, 2008.

[2] Brophy, J. R., Katz, I., Polk, J. E., and Anderson, J. R., "Numerical Simulations of Ion Thruster Accelerator Grid Erosion,” 38th AIAA/ASME/SAE/ASEE Joint Propulsion Conference, Indianapolis, IN, 2002. AIAA-2002-4261.

[3] Anderson, J. R., Katz, I., and Goebel, D., "Numerical Simulations of Two-Grid Ion Optics Using a 3D Code," 40th AIAA/ASME/SAE/ASEE Joint Propulsion Conference, Fort Lauderdale, FL, 2004. AIAA-2004-3782.

[4] Wirz, R. E., Katz, I., Goebel, D. M., and Anderson, J. R., "Electron Backstreaming Determination for Ion Thrusters," Journal of Propulsion and Power, Vol. 27, No. 1, 2011, pp. 206-210.

[5] Wirz, R. E., Anderson, J. R., and Katz, I., “Time-Dependent Erosion of Ion Optics,” Journal of Propulsion and Power, Vol. 27, No. 1, 2011, pp. 211-217.

[6] Bond, R. A., and Latham, P. M., "Ion Thruster Extraction Grid Design and Erosion Modelling using Computer Simulation," 31st AIAA/ASME/SAE/ASEE Joint Propulsion Conference, San Diego, CA, 1995. AIAA-95-2923.

[7] Arakawa, Y., and Nakano, M., "Comparison of Two-Dimensional Optics Codes," 34th AIAA/ASME/SAE/ASEE Joint Propulsion Conference, Cleveland, OH, 1998. AIAA-1998-3801. 
[8] Crofton, M. W., and Boyd, I. D., "Origins of Accelerator Grid Current: Analysis of T5 Grid Test Results," J. Propuls. Power, Vol. 17, No. 1, 2001, pp. 203-211.

[9] Arakawa, Y., and Nakano, M., "An Efficient Three Dimensional Optics Code for Ion Thruster Research," 32nd AIAA/ASME/SAE/ASEE Joint Propulsion Conference, Lake Buena Vista, FL, 1996. AIAA-1996-3198.

[10] Wang, J., Polk, J., Brophy, J., and Katz, I., "Three-Dimensional Particle Simulations of Ion-Optics Plasma Flow and Grid Erosion," J. Propuls. Power, Vol. 19, No. 6, 2003, pp. 1192-1199.

[11] Farnell, C. C., "Performance and Lifetime Simulation of Ion Thruster Optics," Ph.D. thesis, Colorado State University, 2007.

[12] Nakano, M., “Three-Dimensional Simulations of Grid Erosion in Ion Engines,” Vacuum, Vol. 83, 2009, pp. 82-85.

[13] Cao, H., Chu, Y., Wang, E., Cao, Y., Xia, G., and Zhang, Z., "Numerical Simulation Study of Barrel Erosion of Ion Thruster Accelerator Grid,” J. Propuls. Power, Vol. 31, No. 6, 2015, pp. 1785-1792.

[14] Shagayda, A., Nikitin, V., and Tomilin, D., "Three-Dimensional Analysis of Ion Optics with Misalignments of Apertures," Vacuum, Vol. 123, 2016, pp. 140-150.

[15] Shastry, R., Herman, D. A., Soulas, G. C., and Patterson, M. J., "End-of-Test Performance and Wear Characterization of NASA's Evolutionary Xenon Thruster (NEXT) Long-Duration Test," 50th AIAA/ASME/SAE/ASEE Joint Propulsion Conference, Cleveland, OH, 2014. AIAA-2014-3617.

[16] Soulas, G. C., and Shastry, R., "Post-Test Inspection of NASA's Evolutionary Xenon Thruster Long Duration Test Hardware: Ion Optics," 52nd AIAA/SAE/ASEE Joint Propulsion Conference, Salt Lake City, UT, 2016.

[17] Kuzmin, A., Luisier, M., and Schenk, O., "Fast Methods for Computing Selected Elements of the Greens Function in Massively Parallel Nanoelectronic Device Simulations," Euro-Par 2013 Parallel Processing, Lecture Notes in Computer Science, Vol. 8097, edited by F. Wolf, B. Mohr, and D. Mey, Springer, Berlin Heidelberg, 2013, pp. 533-544.

[18] Schenk, O., and Gärtner, K., "Solving Unsymmetric Sparse Systems of Linear Equations with PARDISO," Journal of Future Generation Computer Systems, Vol. 20, No. 3, 2004, pp. 475-487.

[19] Birdsall, C. K., and Langdon, A. B., Plasma Physics Via Computer Simulation, Series in Plasma Physics, Taylor \& Francis, Boca Raton, GL, 2004.

[20] Miller, J. S., Pullins, S. H., Levandier, D. J., Chiu, Y., and Dressler, R. A., "Xenon charge exchange cross sections for electrostatic thruster models," J. Appl. Phys., Vol. 91, No. 3, 2002, pp. 984-991.

[21] Mikellides, I. G., Jongeward, G. A., Katz, I., and Manzella, D. H., "Plume Modeling of Stationary Plasma Thrusters and Interactions with the Express-A Spacecraft," Journal of Spacecraft and Rockets, Vol. 39, No. 6, 2002, pp. 894-903.

[22] LeBeau, G. J., and III, F. E. L., "Application Highlights of the DSMC Analysis Code (DAC) Software for Simulating Rarified Flows," Comput. Methods Appl. Mech. Engrg., Vol. 191, 2001, pp. 595-609.

[23] Araki, S. J., and Wirz, R. E., "Ion-Neutral Collision Modeling Using Classical Scattering with Spin-Orbit Free Interaction Potential,” IEEE Trans. Plasma Sci., Vol. 41, No. 3, 2013, pp. 470-480.

[24] Araki, S. J., and Wirz, R. E., "Modeling of Elastic Collisions between High Energy and Slow Neutral Atoms," 34th International Electric Propulsion Conference, Kobe, Hyogo, Japan, 2015. IEPC-2015-202.

[25] Patino, M. I., and Wirz, R. E., "Characterization of Xenon Ion and Neutral Interactions in a Well-Characterized Experiment," , 2018. Accepted for publication in Phys. Plasmas.

[26] Gibbons, M. R., Kirtley, D. E., VanGilder, D. B., and Fife, J. M., "Flexible Three-Dimensional Modeling of Electric Thrusters in Vacuum Chambers," 39th AIAA/ASME/SAE/ASEE Joint Propulsion Conference, Huntsville, AL, 2003. AIAA-2003-4872.

[27] Yim, J. T., “A Survey of Xenon Ion Sputter Yield Data and Fits Relevant to Electric Propulsion Spacecraft Integration,” 35th International Electric Propulsion Conference, Atlanta, GA, 2017. IEPC-2017-060.

[28] Yalin, A. P., Williams, J. D., Surla, V., and Zoerb, K. A., "Differential Sputter Yield Profiles of Molybdenum Due to Bombardment by Low Energy Xenon Ions at Normal and Oblique Incidence,” J. Phys. D: Appl. Phys., Vol. 40, 2007, pp. 3194-3202. 
[29] Mandell, M. J., Davis, V. A., Pencil, E. J., Patterson, M. J., McEwen, H. K., Foster, J. E., and Snyder, J. S., "Modeling the NEXT Multithruster Array Test with Nascap-2k," IEEE Trans. Plasma Sci., Vol. 36, No. 5, 2008, pp. 2309-2318.

[30] Pollard, J. E., Diamant, K. D., Crofton, M. W., Patterson, M. J., and Soulas, G. C., "Spatially-Resolved Beam Current and Charge-State Distributions for the NEXT Ion Engine," 46th AIAA/ASME/SAE/ASEE Joint Propulsion Conference, Nashville, TN, 2010. AIAA-2010-6779.

[31] Kamhawi, H., Soulas, G. C., Patterson, M. J., and Frandina, M. M., "NEXT Ion Engine 2000 Hour Wear Test Plume and Erosion Results,” 40th AIAA/ASME/SAE/ASEE Joint Propulsion Conference, Fort Lauderdale, FL, 2004. AIAA-2004-3792.

[32] Polk, J. E., Anderson, J. R., Brophy, J. R., Rawlin, V. K., Patterson, M. J., and Sovey, J., "In Situ, Time-Resolved Accelerator Grid Erosion Measurements in the NSTAR 8000 Hour Ion Engine Wear Test," 25th International Electric Propulsion Conference, Cleveland, OH, 1997. IEPC-1997-047. 